\title{
An Optimized High Throughput Clean-Up Method Using Mixed-Mode SPE Plate for the Analysis of Free Arachidonic Acid in Plasma by LC-MS/MS
}

\author{
Wan Wang, ${ }^{1}$ Suzi Qin, ${ }^{2}$ Linsen Li, $^{2}$ Xiaohua Chen, ${ }^{2}$ Qunjie Wang, ${ }^{2}$ and Junfu Wei ${ }^{3}$ \\ ${ }^{1}$ School of Material Science and Engineering, Tianjin Polytechnic University, Tianjin 300387, China \\ ${ }^{2}$ Bonna-Agela Technologies, Tianjin 300462, China \\ ${ }^{3}$ School of Environment \& Chemical Engineering, Tianjin Polytechnic University, Tianjin 300387, China \\ Correspondence should be addressed to Junfu Wei; jfwei@tjpu.edu.cn
}

Received 16 October 2014; Revised 2 December 2014; Accepted 20 December 2014

Academic Editor: Shahram Seidi

Copyright (C) 2015 Wan Wang et al. This is an open access article distributed under the Creative Commons Attribution License, which permits unrestricted use, distribution, and reproduction in any medium, provided the original work is properly cited.

\begin{abstract}
A high throughput sample preparation method was developed utilizing mixed-mode solid phase extraction (SPE) in 96-well plate format for the determination of free arachidonic acid in plasma by LC-MS/MS. Plasma was mixed with $3 \%$ aqueous ammonia and loaded into each well of 96-well plate. After washing with water and methanol sequentially, $3 \%$ of formic acid in acetonitrile was used to elute arachidonic acid. The collected fraction was injected onto a reversed phase column at $30^{\circ} \mathrm{C}$ with mobile phase of acetonitrile/water $(70: 30, \mathrm{v} / \mathrm{v})$ and detected by LC-MS/MS coupled with electrospray ionization (ESI) in multiple reaction monitoring (MRM) mode. The calibration curve ranged from 10 to $2500 \mathrm{ng} / \mathrm{mL}$ with sufficient linearity $\left(r^{2}=0.9999\right)$. The recoveries were in the range of $99.38 \%$ to $103.21 \%$ with RSD less than $6 \%$. The limit of detection is $3 \mathrm{ng} / \mathrm{mL}$.
\end{abstract}

\section{Introduction}

Arachidonic acid is a $\omega-6$ long chain polyunsaturated fatty acid, a senior unsaturated fatty acid. There is high content of free arachidonic acid in the human body which usually comes from dietary animal sources-meat, eggs, and dairyor is converted from linoleic acid. Arachidonic acid and its metabolites have a strong biological activity and can regulate a wide variety of physiological processes, such as the regulation of lipid and glucose, prevention of cardiovascular disease, chemoprevention of cancer cells, and improvement of memory [1-5]. Therefore, it is important to determine the concentration of free arachidonic acid in human plasma for medical research and clinical diagnosis (Figure 1).

There are many methods to detect arachidonic acid in plasma, such as gas chromatography coupled with mass spectrometry, liquid chromatography with precolumn derivation fluorescence detection, and ELISA method [6-10]. However, these methods are either too complicated to operate or lack of good reproducibility. In contrast, LC-MS/MS has been well accepted for the determination of arachidonic acid with high sensitivity [11-17]. In order to detect arachidonic acid in plasma by LC-MS/MS, the samples have to be pretreated to remove interferences which may result in matrix effect on mass spectrometry [18-21]. Although the traditional techniques such as protein precipitation (PPT), liquid-liquid extraction (LLE), and SPE with reversed phase packing material have been reported for clean-up of plasma samples, it is clear that these techniques are insufficient to remove interferences of phospholipids and proteins in plasma [2224], while the combination of ionic interaction and reversed phase interaction was reported to remove phospholipids and proteins more sufficiently in the clean-up process for plasma samples [25]. Furthermore, the analytical chemists in the field of preclinical researches and routine clinical testing are facing thousands of samples. Therefore, it is necessary to establish a simple, fast, efficient, and high throughput sample cleanup method for LC-MS/MS analysis of numerous samples $[26,27]$.

The attention of this study was focused on the development of a high throughput and sufficient sample clean-up 
TABLE 1: MS parameters.

\begin{tabular}{lcccccccccc}
\hline Analyte & $t_{R} / \mathrm{min}$ & $\mathrm{Q} 1$ & $\mathrm{Q} 3$ & $\mathrm{DP}$ & $\mathrm{CE}$ & $\mathrm{IS} / \mathrm{V}$ & $\mathrm{TEM} /{ }^{\circ} \mathrm{C}$ & $\mathrm{GS} 1 / \mathrm{Pa}$ & $\mathrm{GS} 2 / \mathrm{Pa}$ & $\mathrm{CUR} / \mathrm{Pa}$ \\
\hline \multirow{2}{*}{ Arachidonic acid } & 3.3 & 303 & 259.1 & -109 & -18 & -4500 & 500 & 55 & \multirow{2}{*}{35} \\
& & 303 & 205.1 & -107 & -20 & & & & \\
\hline Phospholipids & 4.1 & 496.3 & 184.3 & 63 & 20 & +5500 & 600 & 50 & 50 & 15 \\
\hline
\end{tabular}

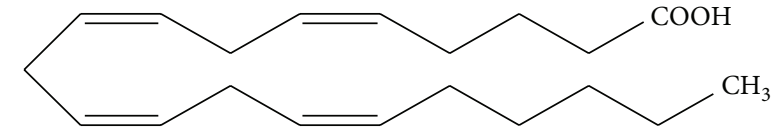

FIgURE 1: Chemical structure of arachidonic acid.

method prior to the analysis of arachidonic acid in plasma by LC-MS/MS. Various sample preparation methods including PPT, LLE, single-mode SPE with nonpolar interaction, and mixed-mode SPE with multiple interaction were studied. The matrix effect of both phospholipids and proteins on the recovery of arachidonic acid was investigated and the final method was applied for the assay of some human plasma samples.

\section{Experimental}

2.1. Materials and Reagents. Arachidonic acid (purity of 99\%), formic acid, and ammonia were purchased from SigmaAldrich (St. Louis, MO, USA). Methanol, acetonitrile, and ethyl acetate were of HPLC-grade and were purchased from Merck (Darmstadt, Germany). Purified water was produced by a Milli-Q Academic System (Millipore, Billerica, MA, USA). Human plasma samples were obtained from local hospital.

Cleanert PPT 96-well plate, Cleanert collection 96-well plate $(2 \mathrm{~mL})$, Cleanert PEP 96-well plate $(60 \mathrm{mg} /$ well $)$, and Cleanert MAS-M 96-well plate $(60 \mathrm{mg} /$ well) were purchased from Agela Technologies (Wilmington, Delaware, USA).

2.2. Instrumentation. Positive pressure SPE manifold, vortex mixer, and centrifuge for 96-well plate were purchased from Agela Technologies (Wilmington, Delaware, USA).

The analysis was accomplished with a Shimadzu LC20A binary HPLC system with an autoinjector coupled with API4000+ triple quadrupole tandem mass spectrometer (AB SCIEX, MA, USA). AB SCIEX Analyst software (version 1.5.1) was used for data acquisition. The HPLC column was a $3 \mu \mathrm{m}$, $2.1 \mathrm{~mm} \times 50 \mathrm{~mm}$ Venusil ASB C18 (Agela Technologies) operated at $30^{\circ} \mathrm{C}$ under an isocratic condition with mobile phase of acetonitrile/water $(75: 25, \mathrm{v} / \mathrm{v})$. Flow rate was $0.2 \mathrm{~mL} / \mathrm{min}$ and injection volume was $5 \mu \mathrm{L}$. The target compounds eluted from the HPLC column were introduced directly into the MS source. Electrospray ionization (ESI) with negative ion mode was selected for arachidonic acid and that with positive ion mode was selected for phospholipids, respectively. Quantitative analysis was performed under MRM mode by calculating the peak areas. The optimal MS parameters were listed in Table 1 . The ions were detected by multiple reaction monitoring (MRM), monitoring the $[\mathrm{M}+\mathrm{H}]^{+}$transition of the $m / z$ precursor ion to the $m / z$ of the product ion

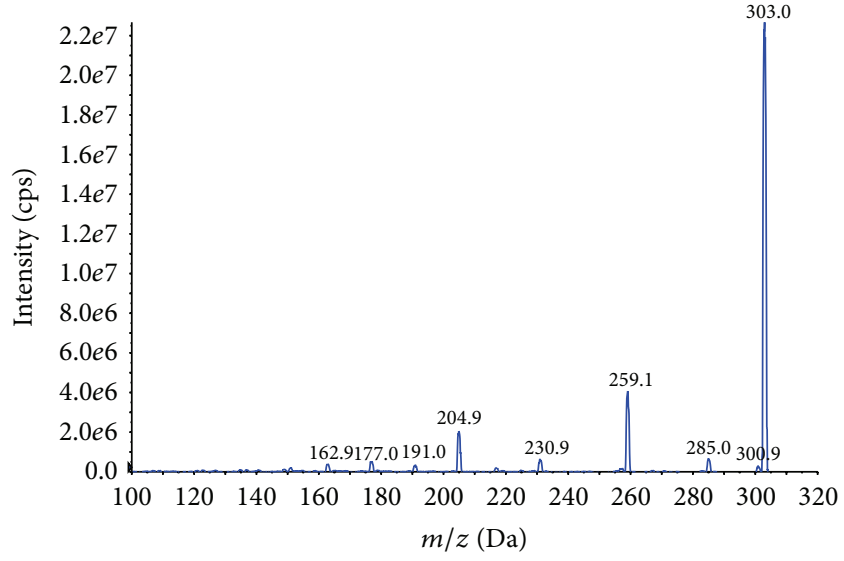

FIgURE 2: Product ion mass spectra of arachidonic acid.

for arachidonic acid. These MS/MS transitions utilized for analysis were $m / z$ 303/259.1 and 303/205.1. An example of the mass spectra of arachidonic acid was shown in Figure 2. A UV detector at $254 \mathrm{~nm}$ wavelength was applied for the detection of proteins.

2.3. Standards and Stock Solutions. $10 \mathrm{mg}$ of arachidonic acid was dissolved in $100 \mathrm{~mL}$ of methanol to prepare a stock solution at $100 \mu \mathrm{g} / \mathrm{mL}$. The stock solution was further diluted with a mixture of acetonitrile: water $(70: 30, \mathrm{v} / \mathrm{v})$ to obtain work solution with the required concentration of arachidonic acid.

2.4. Sample Pretreatments. PPT is a widely adopted sample pretreatment for plasma with routine procedures [22], while LLE and single-mode SPE with reversed phase packing material have been reported to pretreat plasma when arachidonic acid was detected $[11,24]$. They were compared with Cleanert MAS-M which was a mixed-mode SPE to extract arachidonic acid in plasma. All the procedures for each sample pretreatment method were described as follows.

$5 \mathrm{~mL}$ of plasma spiked with $50 \mu \mathrm{L}$ of arachidonic acid work solution was mixed by vortex for 30 seconds to get homogenate samples.

2.4.1. Method A: Protein Precipitation. $100 \mu \mathrm{L}$ of plasma sample diluted with $100 \mu \mathrm{L}$ of $1 \%$ formic acid was loaded into each well of protein precipitation plate followed by $400 \mu \mathrm{L}$ of acetonitrile. The plate was vortexed for $30 \mathrm{sec}$. After centrifuging at $6000 \mathrm{rpm}$ for $5 \mathrm{~min}$, the plate was set on a positive pressure 96-well plate manifold for eluting the target compound into 96-well collection plate. The eluates were dried at $45^{\circ} \mathrm{C}$ under a gentle stream of nitrogen. The residues 
were reconstituted with $200 \mu \mathrm{L}$ of acetonitrile : water $(70: 30$, $\mathrm{v} / \mathrm{v})$ for LC-MS/MS analysis.

2.4.2. Method B: Liquid-Liquid Extraction. $100 \mu \mathrm{L}$ of plasma sample diluted with $100 \mu \mathrm{L}$ of $1 \%$ formic acid was loaded into each well of 96-well collection plate followed by $5 \mu \mathrm{L}$ of methanol. After $30 \mathrm{sec}$ vortex, $500 \mu \mathrm{L}$ of ethyl acetate was added to each well of the plate and then vortexed for $3 \mathrm{~min}$. The plate was stood for $1 \mathrm{~min}$ and centrifuged at $6000 \mathrm{rpm}$ for $5 \mathrm{~min}$. The supernatants were transferred into a clean collection plate sequentially and were dried at $45^{\circ} \mathrm{C}$ under a gentle stream of nitrogen. The residues were reconstituted with $200 \mu \mathrm{L}$ of acetonitrile : water $(70: 30, \mathrm{v} / \mathrm{v})$ for LC-MS/MS analysis.

2.4.3. Method C: Solid Phase Extraction with Cleanert PEP. $100 \mu \mathrm{L}$ of plasma sample diluted with $100 \mu \mathrm{L}$ of $1 \%$ formic acid was loaded into each well of Cleanert PEP, a singlemode SPE plate packed with reversed phase resin. The plate was preconditioned with $1 \mathrm{~mL}$ of methanol and $1 \mathrm{~mL}$ of water sequentially. $500 \mu \mathrm{L}$ of methanol : water $(5: 95, \mathrm{v} / \mathrm{v})$ was used to wash each well of the plate. The target compounds were eluted with $2 \mathrm{~mL}$ of $5 \%$ ammonia in acetonitrile. The eluates were collected into collection plate and further concentrated at $45^{\circ} \mathrm{C}$ under a gentle stream of nitrogen until dryness. The residues were reconstituted with $200 \mu \mathrm{L}$ of acetonitrile : water $(70: 30, \mathrm{v} / \mathrm{v})$ for LC-MS/MS analysis.

2.4.4. Method D: Solid Phase Extraction with Cleanert MASM. $100 \mu \mathrm{L}$ of plasma sample diluted with $100 \mu \mathrm{L}$ of $3 \%$ ammonium hydroxide was loaded into each well of Cleanert MAS-M, a mixed-mode SPE plate which was preconditioned with $1 \mathrm{~mL}$ of methanol and $1 \mathrm{~mL}$ of water sequentially. The plate was washed with $500 \mu \mathrm{L}$ of water followed by $500 \mu \mathrm{L}$ of methanol. The target compound was eluted with $600 \mu \mathrm{L}$ of $3 \%$ formic acid in acetonitrile and collected into collection plate. The eluates were concentrated at $45^{\circ} \mathrm{C}$ under a gentle stream of nitrogen until dryness. The residues were reconstituted with $200 \mu \mathrm{L}$ of acetonitrile : water $(70: 30, \mathrm{v} / \mathrm{v})$ for LC-MS/MS analysis.

\section{Results}

3.1. Comparison of Sample Pretreatment Methods on the Effect of Eliminating Phospholipids. It is well known that phospholipids in plasma will result in matrix effect on mass spectrometry $[19,20]$. Therefore, it is necessary to remove phospholipids from the samples before injection. Although PPT is a common method for biosample preparation [22], the method is unable to remove the phospholipids from plasma efficiently. As shown in Figure 3 (PPT), the eluate obtained from Method A results in a big phospholipids peak that may influence the analysis of arachidonic acid. Although LLE is a widely used sample preparation method to extract the target compounds from aquatic samples [23], the result of Method B (Figure 3 LLE) indicates that a wide peak of phospholipids appeared. Comparing with LLE, SPE has become a popular sample preparation technique in terms of reproducibility,

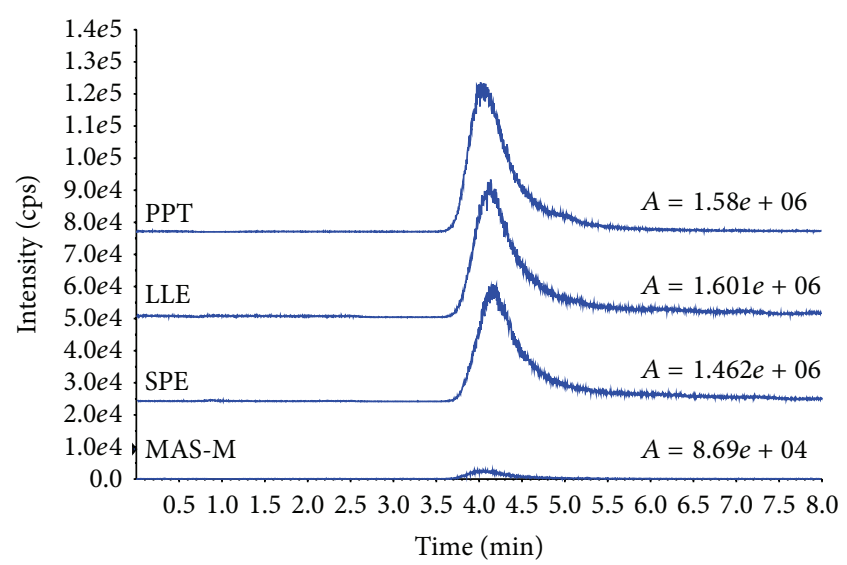

FIGURE 3: Chromatograms and peak areas of phospholipids in plasma treated with various clean-up methods, where " $A$ " = peak area of phospholipids.

less usage of organic solvents, and ease of use. Moreover, SPE is very compatible with an automatic system for high throughput analysis. Arachidonic acid is a hydrophobic compound with Log Kow 7.27; in Method C, therefore, a Cleanert PEP plate packed with nonpolar polymer material was used. However, as shown in Figure 3 (SPE), a broad high peak of phospholipids still remains after SPE clean-up. It is apparent to see from Figure 3 (MAS-M) that Method $\mathrm{D}$ is the best one for removing phospholipids from plasma. Cleanert MAS-M plate is packed with mixed resins with nonpolar, anion exchange, and cation exchange interactions. Since the pKa of arachidonic acid is 4.77 , it is retained on the plate by both anion exchange and nonpolar interactions while the phospholipids and some proteins are retained on the plate by cation exchange and nonpolar interactions under experiment conditions during sample loading. Water soluble interferences are washed out with water and the nonpolar interferences are removed by methanol. Since the arachidonic acid is adsorbed on the plate by anion interaction, there is no loss when the plate is washed by methanol. Finally, after optimizing, $600 \mu \mathrm{L}$ of $3 \%$ formic acid in acetonitrile is applied to release arachidonic acid from the plate while phospholipids with choline groups and proteins with poly amino-groups are retained by the Cleanert MAS plate.

3.2. Comparison of Sample Pretreatment Methods on the Effect of Eliminating Proteins. Proteins with larger molecular weight have similar retention behaviors as arachidonic acid on reversed phase HPLC column. They would cause matrix effect on the determination of arachidonic acid. As shown in Figure 4 (PPT), there are two big peaks that appeared in Method A while the rest of sample pretreatment methods are effective enough to remove proteins from plasma (Figure 4: LLE, SPE, and MAS-M). Combined with the capability for removing phospholipids and proteins, Method D with Cleanert MAS-M plate was selected for further investigation of the analysis of arachidonic acid by LC-MS/MS. 
TABLE 2: Recoveries of arachidonic acid in spiked samples treated by four sample pretreatment methods.

\begin{tabular}{|c|c|c|c|c|c|c|c|c|}
\hline \multirow{2}{*}{$\begin{array}{l}\text { Concentration of arachidonic } \\
\text { acid spiked in plasma }\end{array}$} & \multicolumn{2}{|c|}{$\mathrm{PPT}(n=5)$} & \multicolumn{2}{|c|}{$\operatorname{LLE}(n=5)$} & \multicolumn{2}{|c|}{$\operatorname{SPE}(n=5)$} & \multicolumn{2}{|c|}{ MAS-M $(n=5)$} \\
\hline & Recoveries (\%) & RSD (\%) & Recoveries (\%) & RSD (\%) & Recoveries (\%) & RSD (\%) & Recoveries (\%) & RSD (\%) \\
\hline $500 \mathrm{ng} / \mathrm{mL}$ & 129.32 & 11.14 & 85.48 & 55.90 & 132.95 & 19.60 & 103.21 & 5.17 \\
\hline $2 \mu \mathrm{g} / \mathrm{mL}$ & 130.42 & 2.06 & 67.05 & 82.21 & 94.66 & 9.21 & 99.38 & 5.34 \\
\hline
\end{tabular}



FIGURE 4: Chromatograms of proteins in samples treated with various clean-up methods, where PPT is Method A, LLE is Method $\mathrm{B}, \mathrm{SPE}$ is Method C, and MAS-M is Method D.

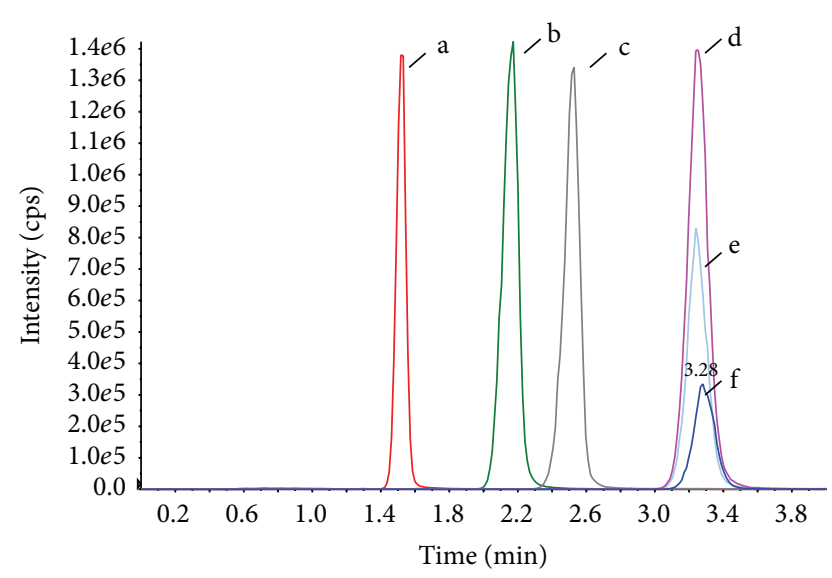

FIGURE 5: Chromatograms of arachidonic acid in different samples. a: unspiked plasma of Method C, b: unspiked plasma of Method B, c: unspiked sample of Method A, d: spiked plasma of Method D at a concentration of $500 \mathrm{ng} / \mathrm{mL}$, e: unspiked plasma of Method D, and f: arachidonic acid standard, $500 \mathrm{ng} / \mathrm{mL}$.

3.3. Comparison Study on the Chromatographic Behaviors and Recoveries of Arachidonic Acid of Various Sample Pretreatment Methods. It is noted that, as shown in Figure 5, the retention time of arachidonic acid in samples treated by Methods A to $\mathrm{C}$ is shifted compared with that of arachidonic acid standard. This phenomenon may be due to the accumulation of residual phospholipids and proteins on reversed phase HPLC column. In contrast, the endogenous interferences are removed efficiently in Method D by Cleanert MAS-M; the retention of arachidonic acid is stable. Also, the results listed in Table 2 reveal the advantage of Cleanert MAS-M.
The recoveries of arachidonic acid by various sample pretreatment methods in two concentration levels are listed in Table 2. As discussed in Sections 3.1 and 3.2, the elimination of phospholipids and proteins is not satisfactory by Method A (PPT), Method B (LLE), and Method C (singlemode SPE with Cleanert PEP). The matrix effect caused by phospholipids and proteins is considered as the main factor to cause the variable recoveries of arachidonic acid. In contrast, Method D with Cleanert MAS-M results in efficient removing of impurities and obtains a sufficient, robust recovery of arachidonic acid.

For quantitation, in order to compare the absolute recoveries of various sample preparation methods, the external standard method is used so that the matrix effect on the absolute recoveries can be observed clearly by contrast with Method D and others.

\subsection{Method Validation}

3.4.1. Linearity, Limits of Detection, and Quantitation. The calibration curve range of $10 \sim 2500 \mathrm{ng} / \mathrm{mL}$ was calculated by a regression analysis of the data to a linear fit with a weighting factor of $1 / x^{2}$ for the ratio of the peak area of arachidonic acid against the nominal concentration. In this range, a linear curve was obtained with correlation coefficients $\left(r^{2}\right)$ better than 0.9999; the result of the linear regression analysis for arachidonic acid is $y=5.18 e+3 x+9.45 e+4\left(r^{2}=0.9999\right)$.

The limit of detection, defined as the concentration giving the signal to noise ratio of 3 , was estimated to be $3 \mathrm{ng} / \mathrm{mL}$ for arachidonic acid.

3.4.2. Precision and Accuracy. The recoveries and precision of the proposed method with Method D are summarized in Table 2. Two concentration levels at $500 \mathrm{ng} / \mathrm{mL}$ and $2 \mu \mathrm{g} / \mathrm{mL}$ were measured. The average recoveries are in the range of $99.38 \% \sim 103.21 \%$ with RSD ranged from $5.17 \%$ to $5.34 \%$.

It is found that adding $100 \mu \mathrm{L}$ of $3 \%$ ammonium hydroxide to dilute plasma is critical to improve the recovery of arachidonic acid, because it will damage the binding of analyte and proteins in plasma by ionizing arachidonic acid. Also, the ionized arachidonic acid will be adsorbed strongly in the loading process by the resin with the combination of anion exchange and nonpolar interaction. By contrast, the recovery of arachidonic acid was insufficient when $100 \mu \mathrm{L}$ of water was used to dilute plasma.

3.5. Applications of the Proposed Methods. The optimized clean-up method using Cleanert MAS-M plate coupled with LC-MS/MS was applied to analyze free arachidonic acid in human plasma samples obtained from local hospital. The results are listed in Table 3. 
TABLE 3: Free arachidonic acid in some human plasma samples.

\begin{tabular}{lrrrrrrrr}
\hline Sample number & 1 & 2 & 3 & 4 & 5 & 6 & 7 & 8 \\
\hline $\begin{array}{l}\text { Concentration } \\
\text { of arachidonic } \\
\text { acid }(\mu \mathrm{g} / \mathrm{mL})\end{array}$ & 1.51 & 0.83 & 1.15 & 0.98 & 1.83 & 2.11 & 2.18 & 1.82 \\
\hline
\end{tabular}

The method was also applied in medical research center of local hospital to determine more than 100 actual plasma samples from patients of coronary heart disease and healthy people. With the high throughput sample clean-up method utilizing 96-well plate, the efficiency of the analysis was improved and well accepted by the researchers from local hospital. The results showed the average concentration of free arachidonic acid in the plasma from patients was lower than that from healthy people, which fit the law of pathology.

\section{Conclusion}

An effective clean-up procedure is developed by comparing four different sample pretreatment methods. The selected method (Method D) is simple, accurate, and precise and has high throughput for the determination of arachidonic acid in plasma samples. In contrast to protein precipitation (Method A), liquid-liquid extraction (Method B), and SPE on Cleanert PEP (Method C), Cleanert MAS-M (Method D) method has better effect on eliminating matrix effect of phospholipids and proteins in the analysis of arachidonic acid in plasma by LC-MS/MS. A sufficient recovery with acceptable precision is reached. The proposed method is successfully applied for determining arachidonic acid in human plasma. The results indicate that the method can be used for routine analysis of arachidonic acid in pharmaceutical industries, hospitals, and research laboratories effectively. Since the 96-well plate was used, the clean-up method can easily be automated. This study has shown the possibility to apply Cleanert MAS-M plate for the pretreatment of hydrophobic analytes in plasma which are usually coeluted with phospholipids and proteins on reversed phase HPLC column.

\section{Conflict of Interests}

All authors declare that there is no conflict of interests in their submitted paper.

\section{Acknowledgments}

This publication is financially supported by the National Key Technology R\&D Program of China (no. 2012BAK25B00) and the National High Technology Research and Development Program of China (no. 2013AA065600).

\section{References}

[1] T. Fukaya, T. Gondaira, Y. Kashiyae et al., "Arachidonic acid preserves hippocampal neuron membrane fluidity in senescent rats," Neurobiology of Aging, vol. 28, no. 8, pp. 1179-1186, 2007.
[2] Z. J. Wang, C. L. Liang, G. M. Li, C. Y. Yu, and M. Yin, "Neuroprotective effects of arachidonic acid against oxidative stress on rat hippocampal slices," Chemico-Biological Interactions, vol. 163, no. 3, pp. 207-217, 2006.

[3] W. S. Harris, D. Mozaffarian, E. Rimm et al., "Omega-6 fatty acids and risk for cardiovascular disease: a science advisory from the American Heart Association nutrition subcommittee of the council on nutrition, physical activity, and metabolism; council on cardiovascular nursing; and council on epidemiology and prevention," Circulation, vol. 119, no. 6, pp. 902-907, 2009.

[4] M. F. Leitzmann, M. J. Stampfer, D. S. Michaud et al., "Dietary intake of n-3 and n-6 fatty acids and the risk of prostate cancer," The American Journal of Clinical Nutrition, vol. 80, no. 1, pp. 204-216, 2004.

[5] G. J. Nelson, P. C. Schmidt, G. Bartolini et al., "The effect of dietary arachidonic acid on plasma lipoprotein distributions, apoproteins, blood lipid levels, and tissue fatty acid composition in humans," Lipids, vol. 32, no. 4, pp. 427-433, 1997.

[6] A. M. Rizzo, G. Montorfano, M. Negroni et al., "A rapid method for determining arachidonic: eicosapentaenoic acid ratios in whole blood lipids: correlation with erythrocyte membrane ratios and validation in a large Italian population of various ages and pathologies," Lipids in Health and Disease, vol. 9, article 7, 2010.

[7] C. M. Zghibeh, V. R. Gopal, C. D. Poff, J. R. Falck, and M. Balazy, "Determination of trans-arachidonic acid isomers in human blood plasma," Analytical Biochemistry, vol. 332, no. 1, pp. 137$144,2004$.

[8] S. Hauff and W. Vetter, "Quantitation of cis- and transmonounsaturated fatty acids in dairy products and cod liver oil by mass spectrometry in the selected ion monitoring mode," Journal of Agricultural and Food Chemistry, vol. 57, no. 9, pp. 3423-3430, 2009.

[9] A. D. Ilyina, J. L. Martinez Hernandez, C. Estrada Badillo et al., "Determination of arachidonic acid based on the prostaglandin $\mathrm{H}$ synthase catalyzed reaction," Applied Biochemistry and Biotechnology_Part A Enzyme Engineering and Biotechnology, vol. 88, no. 1-3, pp. 33-44, 2000.

[10] Y. Arai, T. Fukushima, M. Shirao, X. Yang, and K. Imai, "Sensitive determination of anandamide in rat brain utilizing a coupled-column HPLC with fluorimetric detection," Biomedical Chromatography, vol. 14, no. 2, pp. 118-124, 2000.

[11] T. Y.Zhan, L. Shi, S. J. Zhao et al., "HPLC-MS/MS determination of arachidonic acid in human plasma," Chinese Journal of Pharmaceutical Analysis, vol. 32, no. 3, pp. 388-391, 2012.

[12] L. M. Gonzalez-Reche, A. K. Musiol, A. Müller-Lux, T. Kraus, and T. Göen, "Method optimization and validation for the simultaneous determination of arachidonic acid metabolites in exhaled breath condensate by liquid chromatographyelectrospray ionization tandem mass spectrometry," Journal of Occupational Medicine and Toxicology, vol. 1, article 5, 2006.

[13] Y. Gu, X. Z. Shi, S. M. Zhao, Q. Gu, X. Lu, and G. W. $\mathrm{Xu}$, "Determination of arachidonic acid and its endogenous eicosanoid metabolites in mice tissues using liquid chromatography/electrospray ionization tandem mass spectrometry," Chinese Journal of Analytical Chemistry, vol. 38, no. 8, pp. 10891094, 2010.

[14] D. D. Shinde, K. B. Kim, K. S. Oh et al., "LC-MS/MS for the simultaneous analysis of arachidonic acid and 32 related metabolites in human plasma: basal plasma concentrations and aspirin-induced changes of eicosanoids," Journal of Chromatography B: Analytical Technologies in the Biomedical and Life Sciences, vol. 911, no. 1, pp. 113-121, 2012. 
[15] M. Aslan, F. Özcan, I. Aslan, and G. Yücel, "LC-MS/MS analysis of plasma polyunsaturated fatty acids in type 2 diabetic patients after insulin analog initiation therapy," Lipids in Health and Disease, vol. 12, no. 1, article 169, 2013.

[16] R. C. Murphy, R. M. Barkley, K. Z. Berry et al., "Electrospray ionization and tandem mass spectrometry of eicosanoids," Analytical Biochemistry, vol. 346, no. 1, pp. 1-42, 2005.

[17] D. Tsikas and A. A. Zoerner, "Analysis of eicosanoids by LCMS/MS and GC-MS/MS: a historical retrospect and a discussion," Journal of Chromatography B: Analytical Technologies in the Biomedical and Life Sciences, vol. 964, pp. 79-88, 2014.

[18] R. King, R. Bonfiglio, C. Fernandez-Metzler, C. Miller-Stein, and T. Olah, "Mechanistic investigation of ionization suppression in electrospray ionization," Journal of the American Society for Mass Spectrometry, vol. 11, no. 11, pp. 942-950, 2000.

[19] Y. Q. Xia and M. Jemal, "Phospholipids in liquid chromatography/mass spectrometry bioanalysis: comparison of three tandem mass spectrometric techniques for monitoring plasma phospholipids, the effect of mobile phase composition on phospholipids elution and the association of phospholipids with matrix effects," Rapid Communications in Mass Spectrometry, vol. 23, no. 14, pp. 2125-2138, 2009.

[20] O. A. Ismaiel, M. S. Halquist, M. Y. Elmamly, A. Shalaby, and H. T. Karnes, "Monitoring phospholipids for assessment of matrix effects in a liquid chromatography-tandem mass spectrometry method for hydrocodone and pseudoephedrine in human plasma," Journal of Chromatography B: Analytical Technologies in the Biomedical and Life Sciences, vol. 859, no. 1, pp. 84-93, 2007.

[21] E. Chambers, D. M. Wagrowski-Diehl, Z. Lu, and J. R. Mazzeo, "Systematic and comprehensive strategy for reducing matrix effects in LC/MS/MS analyses," Journal of Chromatography B, vol. 852, no. 1-2, pp. 22-34, 2007.

[22] A. P. Watt, D. Morrison, K. L. Locker, and D. C. Evans, "Higher throughput bioanalysis by automation of a protein precipitation assay using a 96-well format with detection by LC-MS/MS," Analytical Chemistry, vol. 72, no. 5, pp. 979-984, 2000.

[23] C. Kousoulos, G. Tsatsou, C. Apostolou, Y. Dotsikas, and Y. L. Loukas, "Development of a high-throughput method for the determination of itraconazole and its hydroxy metabolite in human plasma, employing automated liquid-liquid extraction based on 96-well format plates and LC/MS/MS," Analytical and Bioanalytical Chemistry, vol. 384, no. 1, pp. 199-207, 2006.

[24] L. Kortz, J. Dorow, S. Becker, J. Thiery, and U. Ceglarek, "Fast liquid chromatography-quadrupole linear ion trap-mass spectrometry analysis of polyunsaturated fatty acids and eicosanoids in human plasma," Journal of Chromatography B: Analytical Technologies in the Biomedical and Life Sciences, vol. 927, pp. 209-213, 2013.

[25] S. T. Wu, D. Schoener, and M. Jemal, "Plasma phospholipids implicated in the matrix effect observed in liquid chromatography/tandem mass spectrometry bioanalysis: evaluation of the use of colloidal silica in combination with divalent or trivalent cations for the selective removal of phospholipids from plasma," Rapid Communications in Mass Spectrometry, vol. 22, no. 18, pp. 2873-2881, 2008.

[26] A. P. Watt, D. Morrison, and D. C. Evans, "Approaches to higherthroughput pharmacokinetics (HTPK) in drug discovery," Drug Discovery Today, vol. 5, no. 1, pp. 17-24, 2000.

[27] N. Zhang, K. Rogers, K. Gajda, J. R. Kagel, and D. T. Rossi, "Integrated sample collection and handling for drug discovery bioanalysis," Journal of Pharmaceutical and Biomedical Analysis, vol. 23, no. 2-3, pp. 551-560, 2000. 

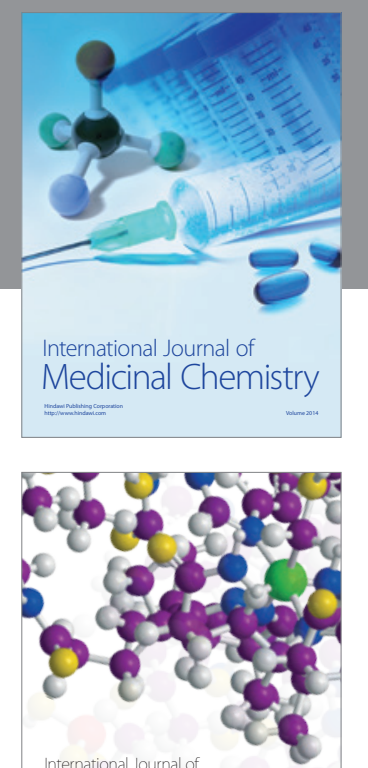

\section{Carbohydrate} Chemistry

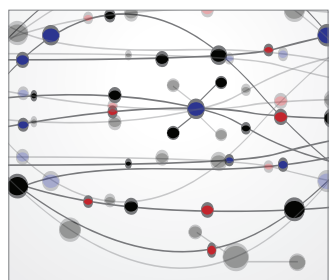

The Scientific World Journal
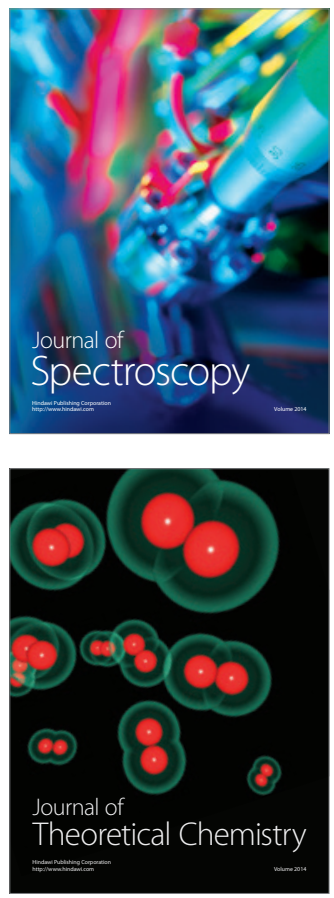
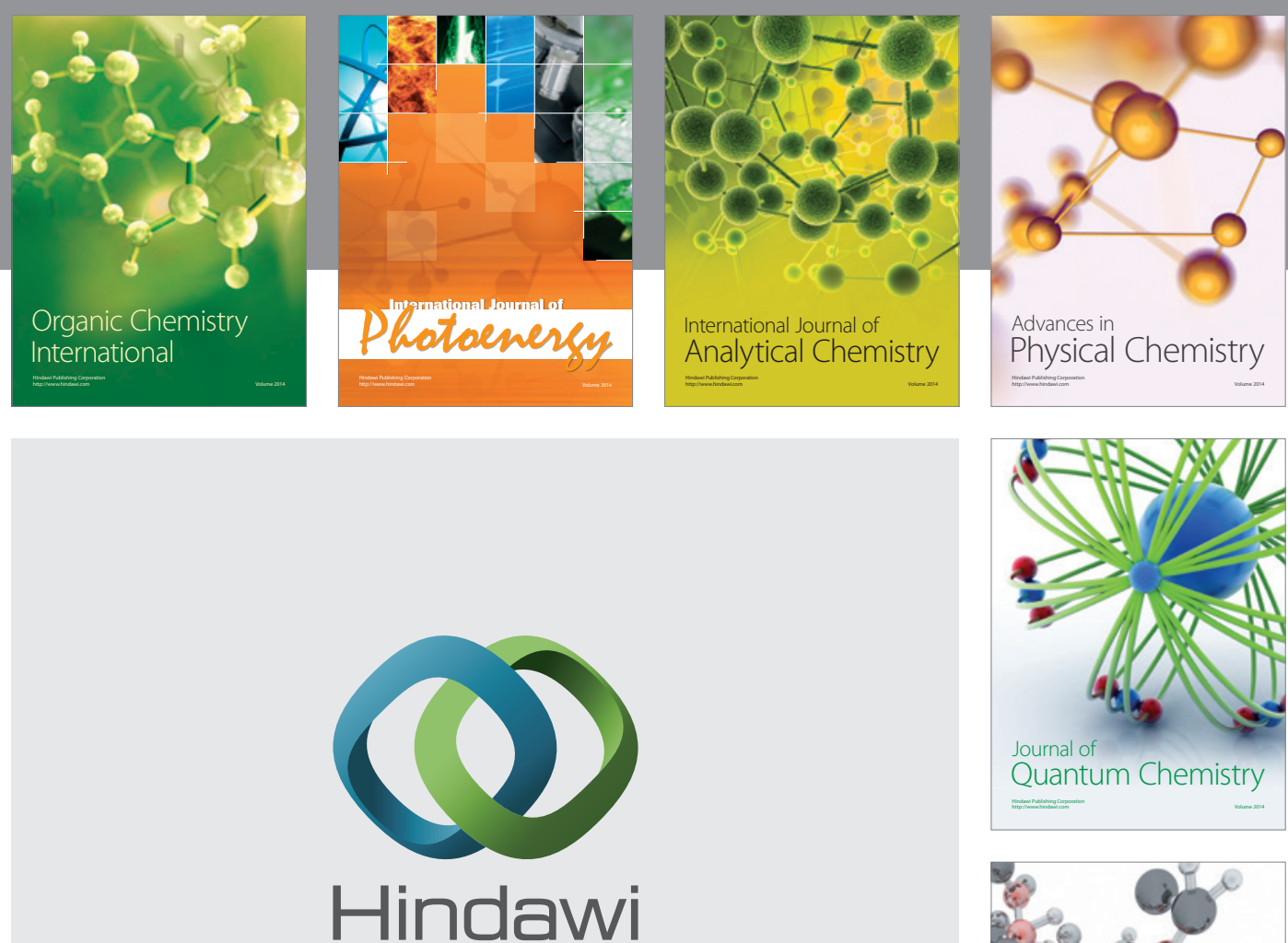

Submit your manuscripts at

http://www.hindawi.com



Analytical Methods

in Chemistry

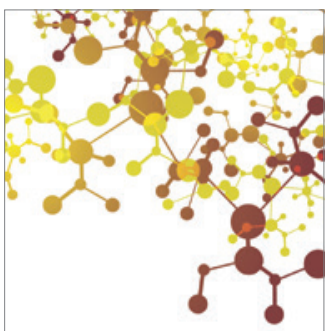

Journal of

Applied Chemistry

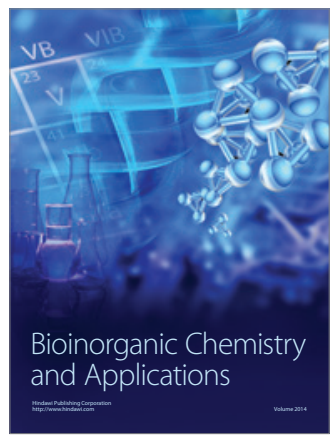

Inorganic Chemistry
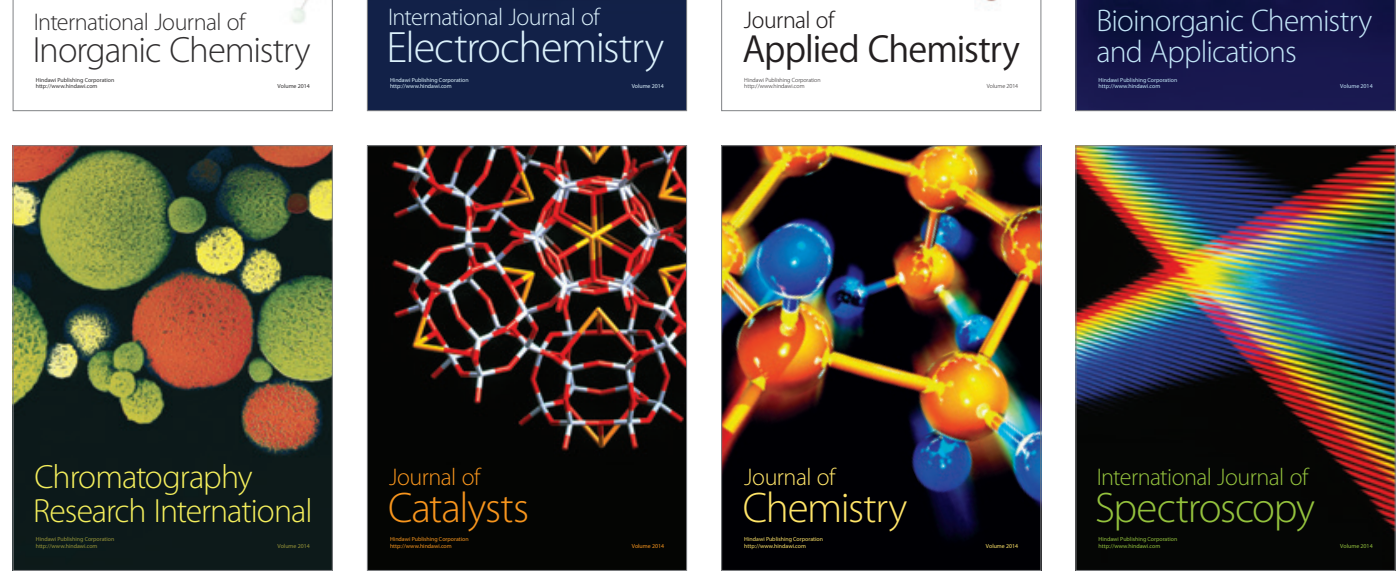